\title{
Gamma-Ray Bursts Observed with the Spectrometer SPI Onboard INTEGRAL
}

\author{
A. von Kienlin*, A. Rau*, V. Beckmann ${ }^{\dagger}$ and S. Deluit** \\ *Max-Planck-Institut für extraterrestrische Physik, Giessenbachstrasse, 85748 Garching, Germany \\ ${ }^{\dagger}$ NASA Goddard Space Flight Center, Greenbelt, Maryland 20771, USA \\ **INTEGRAL Science Data Centre, Chemin d'Écogia 16, 1290 Versoix, Switzerland
}

\begin{abstract}
The spectrometer SPI is one of the main detectors of ESA's INTEGRAL mission. The instrument offers two interesting and valuable capabilities for the detection of the prompt emission of Gamma-ray bursts. Within a field of view of 16 degrees, SPI is able to localize Gamma-ray bursts with an accuracy of 10 arcmin. The large anticoincidence shield, ACS, consisting of $512 \mathrm{~kg}$ of BGO crystals, detects Gamma-ray bursts quasi omnidirectionally above $\sim 70 \mathrm{keV}$. Burst alerts from SPI/ACS are distributed to the interested community via the INTEGRAL Burst Alert System. The ACS data have been implemented into the 3rd Interplanetary Network and have proven valuable for the localization of bursts using the triangulation method. During the first 8 months of the mission approximately one Gamma-ray burst per month was localized within the field of fiew of SPI and 145 Gamma-ray burst candidates were detected by the ACS from which $40 \%$ have been confirmed by other instruments.
\end{abstract}

\section{GRBs observed with the camera of SPI}

The aim of the spectrometer onboard INTEGRAL (SPI) [1] is to perform highresolution spectroscopy of astrophysical sources in the energy range between $20 \mathrm{keV}$ and $8 \mathrm{MeV}$. The imaging capability is good, but is exceeded by that of the imager IBIS which complements SPI by having higher imaging resolution, but lower spectroscopic resolving power. The detection and investigation of cosmic gamma-ray bursts (GRBs) is one of the important scientific topics of the INTEGRAL mission. The broad energy coverage of SPI is well suited to constrain the spectral shape, both below and above the energy at which the GRB power output is typically peaked $(\sim 250 \mathrm{keV})[2]$. The longstanding controversy over the existence of short-lived spectral features in GRB spectra can be addressed by SPI's superb spectroscopic capabilities. In addition, the capability to cross-calibrate both spectra and images between the two experiments is extremely important, particularly in the case of such short-lived events as GRBs, which cannot be re-observed. Currently GRBs which occur inside SPI's field of view (FoV) are detected and analysed offline.

Since the start of the mission, six GRBs have been observed within the FoV of IBIS \& SPI. The obtained scientific results are presented in Malaguti et al. [3] for GRB021125, Mereghetti et al. [4] for GRB 021219, Götz et al. [5] for GRB030131, Mereghetti et al. [6] for GRB0302227, von Kienlin et al. [7] for GRB030320, and Beckmann et al. [8] for GRB030501. In all cases the GRB alert was generated and distributed by IBIS, but SPI was also always able to detect the same event and to confirm in most cases the results 
obtained with IBIS. For the first three bursts the capabilites of SPI were weakened by the telemetry limitations at the beginning of the mission. The third event was the weak GRB030227 and the GRBs of March and May 2003 were observed at a large offset angle, thus only $15 \%$ to $25 \%$ of SPI's Ge-detectors were irradiated by the GRB. So currently the demonstration of SPI's full capabilities in the case of a strong event in the fully-coded FoV still has to take place. An overview on SPI's GRB detection capabilities, obtained for this first set of GRBs is given by von Kienlin et al. [9], which summarises the important quantities derived by SPI.

Most of the GRBs were detected by SPI with a S/N between 7 and 16. At this level the GRBs were located down to error radii of 20' - 30' (90\% confidence) which are in most cases overlapping with the one of IBIS. Also the peak flux, fluence and photon indices are in agreement with the values derived by IBIS. For GRBs observed with SPI operating in full telemetry mode, spectra could be extracted. In one case (GRB030227) some evidence for a hard-to-soft spectral evolution was found in the data of both instruments, ISGRI and SPI [6].

\section{GRBs observed with the anticoincidence shield ACS}

Since December 2002 the anticoincidence shield (ACS) [10] has been added to the $3^{\text {rd }}$ interplanetary network (IPN) of $\gamma$-ray detectors [11]. During the first year of the INTEGRAL mission the IPN consisted of Ulysses, Mars Odyssey 2001, Konus-WIND, HETE-2, RHESSI and INTEGRAL/SPI-ACS. The network had an excellent configuration, due to the large spacecraft separations between Earth, Mars and Ulysses, which is orbiting around the sun, out of the ecliptic plane. The analysis of GRBs detected with the SPI-ACS during the first eight months (November 2002 - June 2003) of the INTEGRAL mission is presented below. A more detailed description of these first results, obtained by SPI-ACS, can be found in von Kienlin et al. [9].

As SPI-ACS has no spatial resolution and thus cannot provide the position of the GRB [10], the GRB nature of a count rate increase observed by SPI-ACS can only be confirmed by the observation of the same event by another instrument (e.g. Ulysses, HETE-2). These bursts constitute only a subsample of the GRBs detected by SPI-ACS, as different instrumental properties of these missions (e.g. energy range, sensitivity) do not allow the simultaneous observations/detections of all GRBs seen by SPI-ACS. Obviously, it is worthwhile to study also bursts which are only visible in the SPI-ACS rates and not confirmed elsewhere. Probing the very high energies with the unprecedented sensitivity of the SPI-ACS [10] might open new insights into the burst populations and burst physics.

A sample of possible GRB events, based on the only property measurable by the SPI-ACS, the veto-countrate lightcurve, is selected for ACS by using only events which exceeds a predefined significance level above the background (the details of the selection procedure are described in von Kienlin et al. [9]). Each event is subsequently checked for solar or particle origin by comparing with events recorded by the X-ray monitor JEM-X and the radiation monitor IREM of INTEGRAL, or events noted on the GOES 

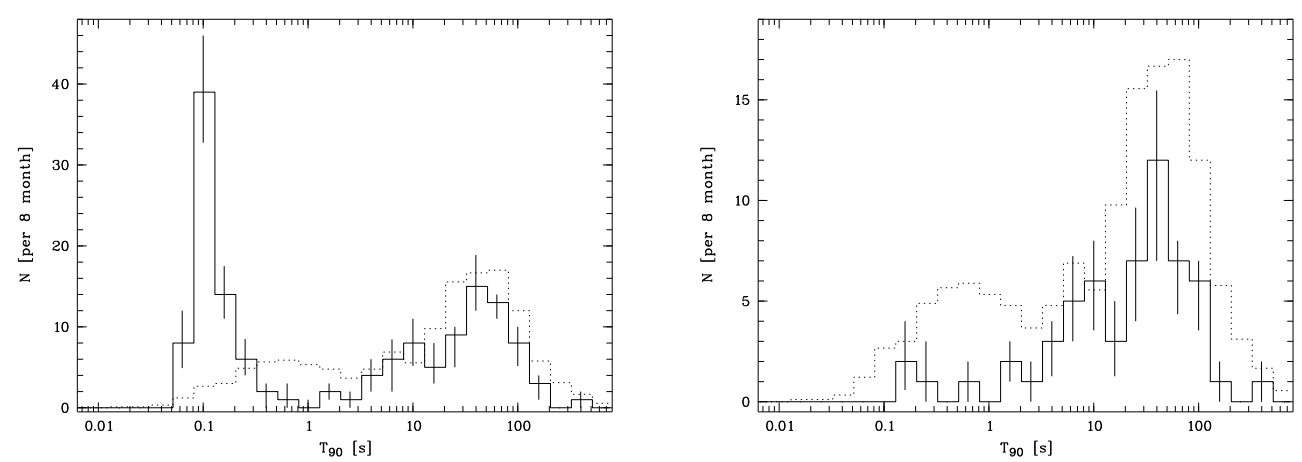

FIGURE 1. Left: Distribution of $\mathrm{T}_{90}$ for all GRB candidates (solid line) and for 1234 GRBs from the 4th BATSE GRB catalogue [13] (dotted). In order to compare with the SPI-ACS detections, the BATSE distribution is scaled to the elapsed INTEGRAL mission time (8 month). Note the very large fraction of short events compared to BATSE. Right: Same as left plot except that here only the confirmed SPI-ACS burst sample (solid line) is displayed. While most of the long duration bursts are confirmed for comparison an obvious lack of short GRBs can be noticed.

web page ${ }^{1}$.

With the selection described above, a total of 145 GRB candidates were detected during the first 8 months of the mission. 58 of these have been confirmed by other instruments. Using the elapsed mission time, we find an approximate rate of GRBs detected by the SPI-ACS of $\sim 290$ ( 116 confirmed) per year which is in good agreement with the predictions given in [12] prior to the start of the mission. The total rate is comparable also to BATSE [13]. In addition to the number of events, the SPI-ACS overall rate provides the possibility of deriving the burst duration in the instrumental observer frame and the variability of the light curve. As no energy resolution exists, typical burst parameters such as fluence and peak flux cannot be derived. Only the total integrated counts and the counts in the burst maximum can be extracted from the light curve. Fig. 11 shows the distribution of the measure for the duration $\mathrm{T}_{90}$ (the time interval starting after $5 \%$ and ending after $95 \%$ of the background subtracted event counts have been observed) for the sample of SPI-ACS GRBs in comparison to the observed distribution of 1234 GRBs from the $4^{\text {th }}$ BATSE GRB catalogue [13]. Despite the small sample, a bimodality in the distribution comparable to that found by BATSE is observed. But two main differences emerge: i) the SPI-ACS sample contains a significantly higher fraction of short burst candidates and ii) the maximum of the short distribution is offset towards shorter duration for the SPI-ACS sample.

The fraction of short $(<1 \mathrm{~s})$ duration GRBs is $\sim 0.48$ (70/145) for the SPI-ACS sample compared to 0.20 for BATSE [13]. As BATSE was observing a softer energy band (50$320 \mathrm{keV}$ ) and was therefore more sensitive to X-ray rich (long) GRBs than SPI-ACS, a larger short/long rate was expected for the ACS sample. What is remarkable is the sharpness of the short distribution around $0.1 \mathrm{~s}$. Due to the limited time resolution of $50 \mathrm{~ms}$ the short end cannot be sufficiently defined and resolved by our data. The offset

1 http://www.sec.noaa.gov 
of the maximum for the short events to smaller $\mathrm{T}_{90}$ might be due to the different energy bands of SPI-ACS and BATSE. An apparently shorter duration is measured as it would be if the bursts would have been observed by BATSE. As $\mathrm{T}_{90}$ depends strongly on the instrumental characteristics and as it is still unclear how this measure connects to the source frame quantity for a given burst, the discrepancies are neither surprising nor do they necessarily trace different burst populations. Still, the connection of the short events with real GRBs is not clear as this population is only marginally observed by other instruments. While a large fraction $(73 \%$; 55/75) of the long bursts are confirmed, less than $6 \%(4 / 70)$ of the short events were observed by other missions. This might be explained at least twofold. On the one hand we might observe a "real" short and very hard GRBs population, which could so far only be detected with SPI-ACS due to its high sensitivity at very high energies. As the current IPN members and HETE-2 are generally more sensitive at lower energies, the detection of a high fraction of unconfirmed short (and possible hard) events would not be surprising. These bursts should then have peak energies above $400 \mathrm{keV}$. On the other hand, a significant contribution to these short events from instrumental effects and/or cosmic ray events cannot be ruled out. A small contribution might also arise from soft gamma-ray repeaters (SGRs). Without localisation SGR bursts cannot be distinguished from short GRBs within SPI-ACS. The issue of origin of the short events is certainly of high interest and needs a more detailed investigation

\section{ACKNOWLEDGMENTS}

The SPI project has been completed under the responsibility and leadership of CNES. We are grateful to ASI, CEA, CNES, DLR, ESA, INTA, NASA and OSTC for support. The SPI/ACS project is supported by the German "Ministerium für Bildung und Forschung" through DLR grant 50.0G.9503.0.

\section{REFERENCES}

1. Vedrenne, G., Roques, J.-P., Schönfelder, V., et al., A\&A, 411, L63-L70 (2003).

2. Preece, R. D., Briggs, M. S., Mallozzi, R. S., et al., ApJS, 126, 19-36 (2000).

3. Malaguti, G., Bazzano, A., Beckmann, V., et al., A\&A, 411, L307-L310 (2003).

4. Mereghetti, S., Götz, D., Beckmann, V., et al., A\&A, 411, L311-L314 (2003).

5. Götz, D., Mereghetti, S., Hurley, K., et al., A\&A, 409, 831-834 (2003).

6. Mereghetti, S., Götz, D., Tiengo, A., et al., ApJ, 590, L73-L77 (2003).

7. von Kienlin, A., Beckmann, V., Covino, S., et al., A\&A, 411, L321-L325 (2003).

8. Beckmann, V., Borkowski, J., Courvoisier, T. J.-L., et al., A\&A, 411, L327-L330 (2003).

9. von Kienlin, A., Beckmann, V., Rau, A., et al., A\&A, 411, L299-L305 (2003).

10. von Kienlin, A., Arend, N., Lichti, G. G., et al., "Gamma-Ray Burst Detection with INTEGRAL/SPI," in X-Ray and Gamma-Ray Telescopes and Instruments for Astronomy, edited by J. E. Truemper and H. D. Tananbaum, 2003, vol. 4851 of Proceedings of the SPIE, pp. 1336-1346.

11. Hurley, K., "The 3rd Interplanetary Network," in American Institute of Physics Conference Series, 2004, p. this issue.

12. Lichti, G. G., Georgii, R., von Kienlin, A., et al., "The $\gamma$-Ray Burst-Detection System of SPI," in American Institute of Physics Conference Series, 2000, pp. 722-+.

13. Paciesas, W. S., Meegan, C. A., Pendleton, G. N., et al., ApJS, 122, 465-495 (1999). 